\title{
What do the Mt. Wilson stars tell us about solar activity?
}

\author{
K.-P. Schröder ${ }^{1}$, M. Mittag ${ }^{2}$, A. Hempelmann² ${ }^{2}$ J. N. González-Pérez ${ }^{2}$, and J. H. M. M. Schmitt ${ }^{2}$ \\ 1 Departamento de Astronomía, Universidad de Guanajuato, Apartado Postal 144, 36000 Guanajuato, Mexico \\ e-mail: kps@astro.ugto.mx \\ ${ }^{2}$ Hamburger Sternwarte, Universität Hamburg, Gojenbergsweg 112, 21029 Hamburg, Germany
}

Received 17 June 2012 / Accepted 16 January 2013

\begin{abstract}
We relate the evolutionary status and mass of the Mt. Wilson project stars with the type and strength of stellar activity as established in decades of monitoring their chromospheric Ca II K line emission. We specifically derive their positions in the Hertzsprung-Russell-diagram (HRD) from HIPPARCos parallaxes and SIMBAD $B-V$ data, considering and correcting for the effects of different individual stellar metallicities, and place different activity groups of the Mt. Wilson stars on a common set of $Z=0.02$ evolution tracks to obtain a quantitative picture of their relative evolutionary status and mass distribution. We find that, first, the downturn in stellar activity does not depend on absolute age but instead decreases with the relative age as stars advance on the main sequence and thus confirm theoretical expectations, while the most active of the irregularly variable stars are found to scatter around the zero-age main-sequence (ZAMS). Moderately active stars, both with clear cycles like the Sun and those without a dominant activity period, populate the 2nd quarter of main-sequence (MS) evolution. Almost inactive stars are mostly in their 3rd quarter of MS evolution and seem to represent stellar analogues of the solar Maunder minimum state. Totally inactive stars are all in the final quarter of their MS evolution and make up for over $70 \%$ of the Mt. Wilson stars that far evolved (the remainders being only weakly active). Most of these are more massive and younger than the Sun. Accordingly, less massive stars did not have enough time to significantly decrease their activity, since they generally evolve more slowly. We find, second, that the Sun is near an apparent upper mass limit for cyclic activity on the MS, because there are no cyclic MS stars much above one solar mass, at least not in the Mt. Wilson sample. Once put in proper perspective with the other Mt. Wilson stars, the Sun indeed ought to be approaching a gradual transition from moderate cyclic activity to a weak, Maunder-minimum-type state, as historic Maunder-type minima seem to indicate already. In addition, the apparent upper mass limit for MS stars to solar-like cyclic activity, not much above one solar mass, is providing dynamo theory with an interesting new challenge.
\end{abstract}

Key words. Sun: chromosphere - Sun: activity - stars: chromospheres - stars: activity - stars: evolution - stars: solar-type

\section{Introduction}

First initiated by O.C. Wilson at the Mt. Wilson Observatory in the 1960s, the chromospheric activity of a large number of solar-type stars was monitored in their Ca II line emission over three decades and then analysed. The observed sample also included the Sun as a star, which was observed without any spatial resolution in reflected light. As a well-known proxy indicator for stellar magnetic activity, long-term variations in the chromospheric $\mathrm{Ca}$ II emission can provide information on the nature and strength of magnetic activity. The Mt. Wilson monitoring programme (cf., Baliunas et al. 1995) specifically demonstrated that the Sun is not the only star with a more or less periodic activity cycle, but rather such periodic behaviour is quite common, albeit not universal, among the Mt. Wilson sample stars.

At the same time, many solar-like stars do not show any periodic activity cycles like the Sun, and there are other forms of variability in stellar activity among such stars. In particular, the very active stars seem to be more or less chaotic in their variations, while in a third group of stars with a very low level of chromospheric activity one observes more or less constant emission levels in these so-called "flat activity" stars in the Mt. Wilson sample.

Recently Schröder et al. (2012) have confirmed a long-held suspicion that these "flat activity" stars appear to mark the "zeropoint" of stellar activity and seem to show only a basal chromospheric flux component (see, e.g., Schrijver 1987; Strassmeier et al. 1994; Pérez Martínez et al. 2011) just like the entirely inactive Sun as observed during the last solar minimum in 2008-2009. Such a minimum, i.e., basal chromospheric emission and an $S$-index level comparable to "flat" apparently nonactive stars, was observed on several occasions during this last unusually low solar minimum, sparking new speculations about the possibility of a recurrence of the famous Maunder minimum (cf., Eddy 1976; Weiss 2010), i.e, the time between $\approx 1645$ to 1715 , with very little, mostly absent solar activity (for a recent reconstruction of the remnant solar Maunder minimum activity, see Nagovitsyn 2007).

The coincidence of the Maunder minimum with the so-called "little ice ages" is the subject of yet another controversial discussion, i.e., to what extent solar activity may act as a climate driver. While variations in total solar irradiance (TSI) do correlate with solar activity, the overall TSI changes are quite small (e.g., Fröhlich 2011). However, activity-induced flux changes at shorter wavelengths are much larger and, according to Ineson et al. (2011), the solar UV flux may play a rôle, at least locally, in the strength of northern-hemisphere winters; there may be other indirect, positive feedback mechanisms at work, for example, changes in cloud cover (Svensmark 1998) or in the transparency of the Earth's atmosphere (Weber 2010; Hempelmann \& Weber 2011 ), triggered by an activity-controlled variation in the cosmic ray flux.

The question of how frequently Maunder minimum states occur in solar-like stars and how long a Maunder minimum type near-absence of solar activity is to be expected, appears to also 
be of some interest for the modelling of our climate future. To that effect Baliunas et al. (1995) noted that the apparently inactive ("flat") solar-type stars account for about one third of their sample, in later studies this number was revised downwards Baliunas et al. (1998), and other authors (for example Hall \& Lockwood 2004) discuss in detail how stellar analogues of the Maunder minimum can be reliably found. The question remains whether we can then expect such an inactive state for the Sun for, say, one third or one sixth of the time, on average?

The crucial question in this context is clearly which stars can be considered comparable to the Sun and which should be discounted. The Mt. Wilson sample mainly consists of solar-type, main-sequence (MS) stars. Using the coronal X-ray flux rather than the chromospheric CaII $\mathrm{K}$ emission, Hempelmann et al. (1996) confirmed that the "flat", low-S stars are indeed the least active class, the cyclically active stars are intermediate, and the most active class in the Mt. Wilson monitoring project with the largest $S$-indices contains often irregularly variable stars.

Stellar activity is known to be strongly correlated with stellar rotation (Skumanich 1972; Pallavichini et al. 1981), and in turn, stellar rotation strongly depends on age and/or evolutionary stage. Both, rotation rate and stellar activity were shown to decline with age by comparing solar-type stars in different open clusters (see, e.g. Stauffer et al. 1984, 1985; Simon 1990, 1992) and for chromospheric activity in a large sample of stars (Wright et al. 2004).

Studies of the coronal X-ray emission of nearby giant stars (e.g. Hünsch et al. 1996; Hünsch \& Schröder 1996) have suggested a dependence of stellar activity on evolutionary phase and history. More recently, Reiners \& Mohanty (2012) have found the lifetime of angular momentum, and hence stellar activity, on the MS to be proportional to $\left(R^{16} / M^{2}\right)^{-1 / 3}$. With the approximate relation between radius and mass for moderate mass MS stars, $R \propto M^{0.7}$, this factor becomes approximately $\propto M^{-3}$, much like the respective MS lifetime itself. As a result we conclude that stellar activity in a sample of MS stars covering some range of masses should decline with relative MS age, but not with absolute age. In either case, the one or the other quantity should be an additional criterion for any comparison between the Mount Wilson sample stars and the Sun.

Until the availability of precise HIPPARCos parallaxes (Perryman 2002) it was impossible to extract further information especially on the evolutionary states and ages of the Mt. Wilson stars. Using the parallax information from HIPPARCos Wright (2004) could indeed demonstrate that the Maunder minimum stars in the Mt. Wilson sample are indeed all quite evolved, however, the effects of metalicity on the position of a star in the Hertzsprung-Russell diagram (HRD) could not be accounted for. Only quite recently have precise chemical abundances become available for most of these stars (Holmberg et al. 2009), making it possible to provide a more reliable assessment of how much of the respective MS lifetime has already passed for each of the Mt. Wilson stars and in particular assess these stars' relative MS age.

To address the open questions posed above, we here revisit the well-studied Mt. Wilson sample stars both from an observational and a theoretical point of view. Some of these stars have been followed up for another 15 years beyond the original Mt. Wilson campaign as part of other monitoring programmes with very similar motivation (Hall et al. 2007; Lockwood et al. 2007), comparing new $\mathrm{Ca}$ II $\mathrm{K}$ emission line based activity parameters with a calibrated Mt. Wilson $S$-index. Using the Hamburg robotic telescope (HRT) and its HEROS spectrograph, we can now add further $\mathrm{Ca}$ II $\mathrm{K}$ observations obtained in most recent years and demonstrate that we can provide reliable $S$-index values. For this direct comparison, we use our own calibrated Mt. Wilson $S$-index. This way, the non-active state of nine Mt. Wilson "flat" stars can already be confirmed (see below and Table 1, our $S$ values agree within the uncertainties with those of Baliunas et al. (1995), extending the temporal base for these stars to almost 50 years.

The main body of this work, however, is the analysis of the evolutionary stages, relative MS ages, and masses of all Mt. Wilson stars in the colour range of $0.4<B-V<1.0$. In grouping these stars according to different activity types, we compare evolutionary state (i.e., age, relative MS-age and stellar mass) with the respective activity (see Sects. 3 and 4).

\section{New Ca II observations and stellar data}

In our initial observing period at Hamburg Observatory we used the Hamburg robotic telescope (HRT) with its HEROS spectrograph to revisit a number of inactive stars, i.e., nine "flat activity" stars from the Mt. Wilson project and, in addition, the star 15 LMi (from Duncan et al. 1991) and $\eta$ Boo (see Table 1). For a discussion and brief description of the calibration procedure see Schröder et al. (2012), a description of the telescope (HRT) and spectrograph (HEROS) is provided by Hempelmann et al. (2005) and Mittag et al. (2010).

These new observations extend the known timespan of inactivity or low activity of several solar-like stars (see Table 1) by almost two decades, given that the last larger published compilations of Mt. Wilson data are presented by Duncan et al. (1991) and Baliunas et al. (1995). We note that a limited set of time series from the last decade of the Mt. Wilson programme is presented by Lockwood et al. (2007), until the Mt. Wilson programme officially ended in 2003, and that other synoptic programs have only partially overlapped the Mt. Wilson stellar sample.

For the other two stars, i.e, $\eta$ Boo and $15 \mathrm{LMi}$, the low level of activity as shown through their low $S$-levels has been confirmed in the present epoch. In all cases, the calibrated $S$-value obtained with HRT/HEROS agrees very well with the Mt. Wilson values to within the estimated uncertainties. This test has proven our ability to monitor, now and in the future, stellar activity fully consistent with the Mt. Wilson observations. This will be a major key project of our observing programme at the new HRT site in Guanajuato in central Mexico.

As described in more detail by Schröder et al. (2012), the inactive nature of a star with only a basal chromospheric flux is related to a minimal $S$-value that depends, however, on the colour (and thus temperature) and gravity of each star. At some periods during the exceptional 2008/2009 solar minimum, an entirely quiet Sun also reached its minimal $S$-value near 0.156 . The even lower $S$-values listed in Table 1 are mostly from stars with lower-than-solar gravity; and these agree within the observational errors with their respective minimum $S$-values.

\section{Evolution tracks and metallicity effects in the observational HRD}

We now proceed to demonstrate the evolved, late-MS nature of most of these stars by determining their relative MS ages using well-tested evolutionary tracks. For the necessary assessment of the basic stellar parameters we used the SIMBAD database for the observed $B-V$ colours and $V$ magnitudes as well as the HIPPARCos parallaxes, from which the distance modulus and absolute magnitudes $M_{v}$ were obtained. Since the vast majority of 


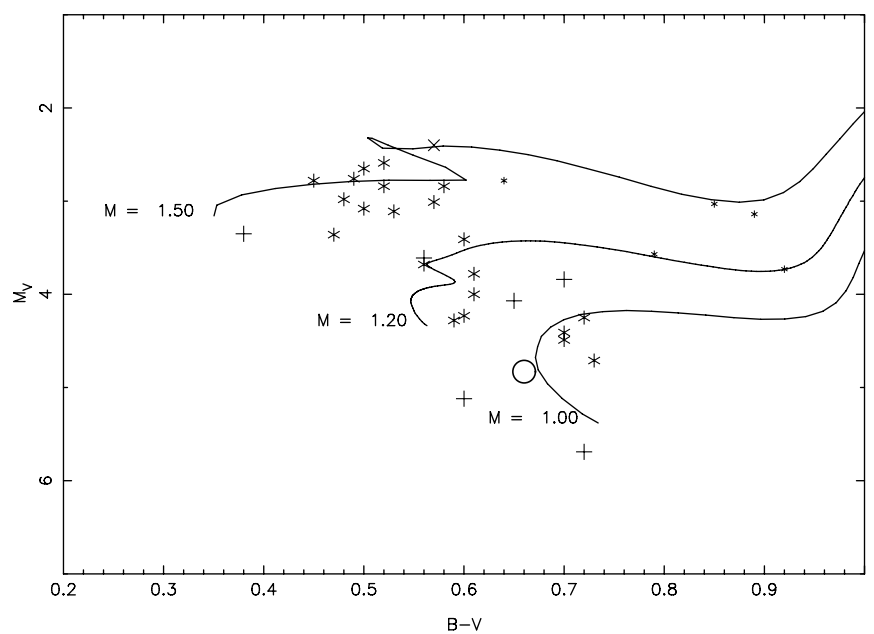

Fig. 1. Raw positions, uncorrected for metallicity, of the inactive ("flat") Mt. Wilson stars in the observational HRD, together with a set of evolutionary tracks, readily revealing these stars their late MS evolutionary stage even by this crude comparison.

Table 1. Stars confirmed or found to be inactive by recent $S$-measurements of HRT/HEROS*, 2008/2009.

\begin{tabular}{lrrrrr}
\hline \hline Star & Name & $B-V$ & $S_{\text {MW }}$ & $S_{\text {HRT }}$ & $\pm \sigma$ \\
\hline HD 13421 & 64 Cet & 0.52 & 0.131 & 0.135 & 0.006 \\
HD 32923 & 104 Tau & 0.65 & 0.143 & 0.140 & 0.006 \\
HD 43587 & & 0.59 & 0.156 & 0.150 & 0.007 \\
HD 84737 & $15 \mathrm{LMi}$ & 0.61 & 0.145 & 0.140 & 0.005 \\
HD 89744 & & 0.49 & 0.137 & 0.141 & 0.005 \\
HD 121370 & $\eta$ Boo & 0.57 & - & 0.132 & 0.005 \\
HD 124570 & 14 Boo & 0.58 & 0.133 & 0.133 & 0.006 \\
HD 142373 & $\chi$ Her & 0.56 & 0.147 & 0.145 & 0.005 \\
HD 207978 & 15 Peg & 0.38 & 0.152 & 0.148 & 0.006 \\
HD 217014 & 51 Peg & 0.70 & 0.149 & 0.144 & 0.007 \\
\hline
\end{tabular}

Notes. ${ }^{(*)}$ Observations carried out at the Hamburger Sternwarte, GER.

the sample stars are at distances much closer than $50 \mathrm{pc}$, interstellar absorption and extinction effects are much less than other uncertainties and were thus neglected.

Already with these basic data, i.e., without considering any metallicity effects on the position in the HRD, the observed HRD suggests that these inactive stars are all evolved far on the MS (see Fig. 1). This relation between inactive stars and evolutionary status has already been noticed and demonstrated by Wright (2004). However, for a quantitative analysis, metallicity must also be considered: As is well known, for given mass and evolutionary age a metal-poor star appears bluer and more luminous than a more metal-rich comparison star. For this reason, the proper assessment of a star's HRD position requires that metallicity effects are accounted for in a quantitative way.

To properly account for stellar metallicity, we adopted the $[\mathrm{Fe} / \mathrm{H}]$-values obtained by Holmberg et al. (2009) from the Strömgren colours of the "Geneva-Copenhagen Survey of the Solar Neighbourhood". To evaluate the evolutionary stage and specifically the relative evolutionary MS-age of the Mt. Wilson stars, we used $Z=0.02$ evolutionary tracks computed with the well-tested and fast Eggleton stellar evolution code (see Pols et al. 1997, and references therein); in particular, the convection parameters and the He content (depending on metallicity) have been well-tested and are very reliably adjusted to match empirical constraints, using not only open clusters, but also precisely known eclipsing binaries with MS and giant components (see Pols et al. 1997; Schröder et al. 1997).

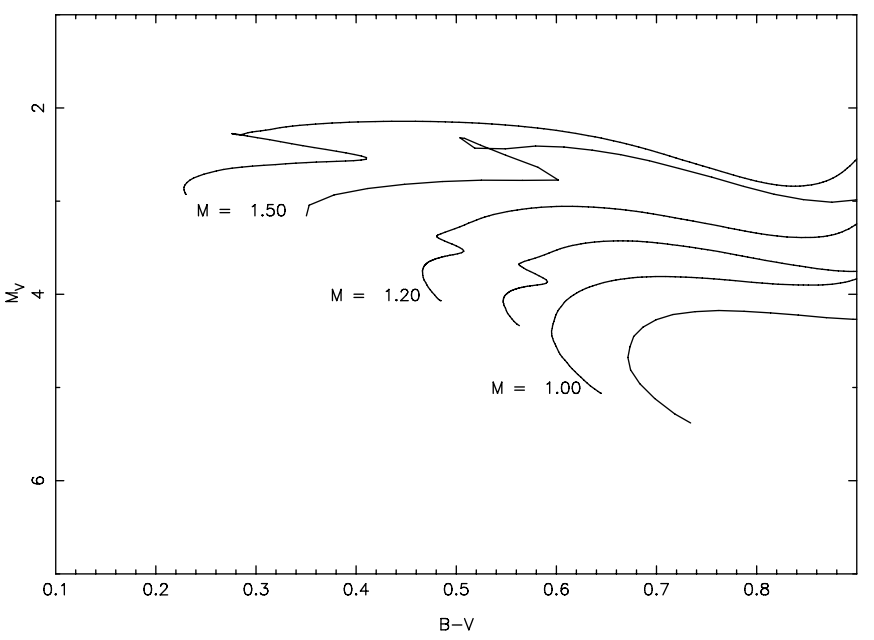

Fig. 2. Comparison of three evolutionary tracks (for stars with 1.0, 1.2, and 1.5 solar masses), computed for metallicities of $Z=0.02$ with those of $Z=0.01$ (the left track of each pair) yields the position shifts used to project the stellar sample onto a common set of evolutionary tracks with $Z=0.02$, as for Figs. 3 to 5).

A comparison with more recent evolution models based on a different evolutionary code (Pietrinferni et al. 2004, 2006) shows very good agreement for tracks with $Z=0.02$ and 0.01 . Pietrinferni et al. use very similar input physics, but make slightly different choices for the He-content $(\Delta Y / \Delta Z=1.5$, instead of $\Delta Y / \Delta Z=2$ as in our work), the mixing length and (enhanced) $\mathrm{CNO}$-abundances, which apparently compensate each other for their effect on the locations of the tracks in the HRD, when compared to our tracks. However, there is a small difference, which is of some relevance for the mass range discussed in this paper. We used a relatively steep onset of "overshooting" between 1.4 and $1.5 M_{\odot}$, confirmed by evidence from both cluster isochrones and from comparison of MS star counts with population models (Schröder \& Pagel 2003), consistent with simple theoretical expectations that "overshooting" (i.e., extended mixing, reaching beyond the Schwarzschild boundary) requires a core with well-developed convection (Roxburgh 1992). Pietrinferni et al., nevertheless use a very smooth onset of overshooting for their model grid, ranging from 1.1 to $1.7 M_{\odot}$, but admit that their own tests with M 67 and AI Phe suggest instead a steep onset of overshooting near $1.3 M_{\odot}$ (Pietrinferni et al. 2004), which in fact is close to our choice.

For convenience, effective temperature and luminosity of the evolution tracks are converted here into $B-V$ and $M_{v}$, using bolometric corrections and colours given by the standard ATLAS models, allowing us to directly compare our models with observed stellar quantities. To quantify the above-mentioned shift of the HRD position caused by a different metallicity, we now compare, for the same relative MS age, evolution tracks with $Z=0.02$ to those computed assuming half the metallicity $(Z=$ $0.01)$. This gives us a range of metallicity of $\Delta[\mathrm{Fe} / \mathrm{H}]=-0.3$ in the relevant mass range of around 1 to $1.5 M_{\odot}$ (see Fig. 2). Over the MS, these shifts in $B-V$ colour and $M_{v}$ are nearly the same and are almost independent of mass: we find shifts of +0.09 in $B-V$ and +0.29 in $M_{v}$ for $0.8 M_{\odot},+0.08$ in $\mathrm{B}-\mathrm{V}$ and +0.26 in $M_{v}$ for $1.0 M_{\odot},+0.08(B-V)$ and $+0.25\left(M_{v}\right)$ for $1.2 M_{\odot}$, $+0.12(B-V)$ and $+0.23\left(M_{v}\right)$ for $1.5 M_{\odot}$. Within about $10 \%$ of uncertainty, these shifts agree with what we can derive from some tracks of Pietrinferni et al. (2006, their Fig. 1) with these parameters. 
Table 2. List of the inactive stars with $S \leq 0.17^{* *}$.

\begin{tabular}{|c|c|c|c|c|c|c|c|}
\hline Star & $B-V$ & $M_{v}$ & {$[\mathrm{Fe} / \mathrm{H}]$} & {$[B-V]^{\prime}$} & $M_{v}^{\prime}$ & Type & $\langle S\rangle$ \\
\hline HD 207978 & 0.38 & 3.35 & -0.51 & 0.55 & 3.80 & flat & 0.152 \\
\hline HD 76572 & 0.42 & 2.53 & -0.34 & 0.53 & 2.85 & poor & 0.148 \\
\hline HD 159332 & 0.45 & 2.78 & -0.19 & 0.55 & 2.96 & long & 0.144 \\
\hline HD 187013 & 0.47 & 3.36 & -0.11 & 0.52 & 3.48 & long & 0.154 \\
\hline HD 216385 & 48 & 2.98 & -0.17 & 0.57 & 3.14 & long & 0.142 \\
\hline HD 89744 & 49 & 2.76 & +0.18 & 0.44 & 2.57 & flat & 0.137 \\
\hline HD 136202 & 50 & 3.08 & +0.03 & 0.51 & 3.08 & long & 0.140 \\
\hline HD 107213 & 0.50 & 2.65 & +0.09 & 0.48 & 2.60 & long & 0.135 \\
\hline HD 212754 & 0.52 & 2.84 & +0.10 & 0.50 & 2.79 & long & 0.140 \\
\hline HD 13421 & 0.52 & 2.59 & +0.11 & 0.50 & 2.54 & flat & 0.131 \\
\hline HD 45067 & 0.53 & 3.11 & -0.08 & 0.58 & 3.24 & flat & 0.141 \\
\hline HD 187691 & 0.56 & 3.68 & +0.10 & 0.55 & 3.63 & $5 ?$ & 0.148 \\
\hline HD 142373 & 0.56 & 3.61 & -0.50 & 0.70 & 4.03 & flat & 0.147 \\
\hline HD 121370 & .57 & 2.40 & +0.27 & 0.49 & 2.23 & - & 0.132 \\
\hline HD 29645 & 57 & 3.01 & +0.14 & 0.54 & 2.93 & long & 0.140 \\
\hline HD 1 & .58 & 2.84 & +0.07 & 0.57 & 2.82 & flat & 0.133 \\
\hline HD 43587 & .59 & 4.28 & -0.11 & 0.63 & 4.40 & flat? & 0.156 \\
\hline 6361 & 60 & 4.23 & -0.25 & 0.68 & 4.47 & long & 0.150 \\
\hline HD 1 & 60 & 5.12 & -0.39 & 72 & 5.47 & cyc? & 0.165 \\
\hline $\mathrm{HD}$ & 60 & 3.41 & +0.13 & 0.57 & 3.33 & long & 0.136 \\
\hline HD 1 & .61 & 4.00 & -0.01 & 0.63 & 4.04 & long & 0.155 \\
\hline HD 84737 & 0.61 & 3.78 & +0.07 & 0.60 & 3.76 & - & 0.137 \\
\hline HD $161239^{*}$ & 0.64 & 2.78 & $\mathrm{n} / \mathrm{a}$ & 0.66 & 2.80 & $5 ?$ & 0.138 \\
\hline HD 32923 & 0.65 & 4.07 & -0.22 & 0.73 & 4.29 & - & 0.143 \\
\hline Sun & 0.66 & 4.83 & -0.04 & 0.68 & 4.87 & cyc & $>0.156$ \\
\hline HD 178428 & 0.70 & 4.41 & +0.05 & 0.70 & 4.40 & flat & 0.154 \\
\hline HD 217014 & 0.70 & 4.49 & +0.12 & 0.68 & 4.42 & var & 0.149 \\
\hline HD 3795 & .70 & 3.84 & -0.42 & 0.83 & 4.24 & var & 0.156 \\
\hline HD 10700 & .72 & 5.69 & -0.42 & 0.91 & 6.09 & flat & 0.171 \\
\hline HD 158614 & .72 & 4.25 & -0.01 & 0.74 & 4.29 & flat & 0.158 \\
\hline HD 190360 & 0.73 & 4.71 & $\mathrm{n} / \mathrm{a}$ & 0.75 & 4.75 & flat & 0.146 \\
\hline HD 219834A* & 0.79 & 3.57 & $\mathrm{n} / \mathrm{a}$ & 0.81 & 3.61 & $21 ?$ & 0.155 \\
\hline HD $188512^{*}$ & 0.85 & 3.03 & $\mathrm{n} / \mathrm{a}$ & 0.87 & 3.07 & poor & 0.136 \\
\hline HD $22072^{*}$ & 0.89 & 3.14 & $\mathrm{n} / \mathrm{a}$ & 0.91 & 3.18 & var & 0.131 \\
\hline HD $23249^{*}$ & 0.92 & 3.73 & $\mathrm{n} / \mathrm{a}$ & 0.94 & 3.77 & flat? & 0.137 \\
\hline
\end{tabular}

Notes. ${ }^{(*)}$ Subgiant or post-MS star. ${ }^{(*)}$ Arranged by observed $B-V$, with their average Mt. Wilson $S$-values, possible periodicity given in years in column "type", according to (Baliunas et al. 1995).

Because of this we can map each star with its measured individual metallicity onto a set of isochrones with $Z=0.02$ by simply shifting it in colour and absolute luminosity correspondingly, interpolating the above shifts over mass whenever necessary. There is only one cyclic active star, viz. HD 103095, which falls outside the mass and metallicity range specified above, so it was not been considered any further. In addition, we excluded those stars from further study, which have evidently already left the MS (notably, five stars in our inactive sample).

We also kept in mind that the Sun has a metallicity of $Z=0.018$ (on the common scale set by using non-HD photospheric model atmospheres), equivalent to a common shift of $[\mathrm{Fe} / \mathrm{H}]=-0.04$ against our standard evolution track metallicity of $Z=0.02$. In addition we applied a small $[B-V]$-scale correction of +0.01 to make the Sun meet its track position exactly. This procedure led us to fiducial $[B-V]^{\prime}$ and $M_{v}^{\prime}$ values, which provide the HRD-position of a star of $Z=0.02$ with the same mass and same relative (evolutionary) MS-age as the original star. In a way, each star with its nominal HRD-position and relative $\mathrm{MS}$ age is projected onto a modified HRD-diagram assuming a metallicity of $Z=0.02$ (see $[B-V]^{\prime}$ and $M_{v}^{\prime}$ in Tables 2 and 3 , below).

In this way, the effects of stellar metallicity can be separated from the evaluation of stellar MS-evolution in the HRD, and we
Table 3. List of the active Mt. Wilson project stars** with clearly cyclic behaviour.

\begin{tabular}{lrrrrrrr}
\hline \hline Star & $B-V$ & $M_{v}$ & {$[\mathrm{Fe} / \mathrm{H}]$} & {$[B-V]^{\prime}$} & $M_{v}^{\prime}$ & $\mathrm{P} / \mathrm{yrs}$ & $S<$ \\
\hline HD 18256 & 0.43 & 2.89 & -0.12 & 0.50 & 3.01 & 6.8 & 0.21 \\
HD 114710 & 0.58 & 4.44 & -0.21 & 0.66 & 4.65 & 10 & 0.23 \\
HD 81809* & 0.62 & 2.98 & -0.29 & 0.75 & 3.24 & 8.2 & 0.20 \\
HD 78366 & 0.63 & 4.48 & -0.10 & 0.68 & 4.60 & 12 & 0.29 \\
Sun & 0.66 & 4.83 & -0.04 & 0.68 & 4.87 & 11 & 0.21 \\
HD 103095 & 0.75 & 6.66 & $-1.36^{+}$ & $1.1:$ & $7.8:$ & 7.3 & 0.22 \\
HD 152391 & 0.76 & 5.46 & -0.08 & 0.81 & 5.56 & var & 0.40 \\
HD 185144 & 0.78 & 5.88 & -0.24 & 0.86 & 6.12 & $7 ?$ & 0.25 \\
HD 26965 & 0.82 & 5.92 & -0.04 & 0.85 & 5.99 & 10 & 0.25 \\
HD 10476 & 0.84 & 5.82 & -0.04 & 0.87 & 5.89 & 9.6 & 0.25 \\
HD 3651 & 0.84 & 5.66 & +0.19 & 0.80 & 5.53 & 14 & 0.23 \\
HD 4628 & 0.89 & 6.38 & -0.17 & 0.95 & 6.56 & 8.4 & 0.30 \\
HD 166620 & 0.90 & 6.19 & -0.08 & 0.94 & 6.29 & 16 & 0.23 \\
HD 219834B & 0.94 & 5.35 & $\mathrm{n} / \mathrm{a}$ & 0.96 & 5.37 & 10 & 0.25 \\
HD 160346 & 0.96 & 6.31 & -0.09 & 1.00 & 6.42 & 7.0 & 0.4 \\
HD 16160 & 0.98 & 6.55 & +0.08 & 0.98 & 6.52 & 13 & 0.29 \\
\hline
\end{tabular}

Notes. ${ }^{(*)}$ Post-MS star +) Pop.II?, ca. $0.6 M_{\odot} \cdot{ }^{(* *)}$ In order by observed $B-V$; activity strength is indicated by the average Mt. Wilson $S$-value in cycle maximum, the dominant periods are given in years, according to Baliunas et al. (1995).

can now compare all stars simultaneously with the same standard set of evolutionary tracks (see Figs. 3-5). We estimated the remaining uncertainties, including the impact from metallicity uncertainties and some low interstellar extinction and absorption, to be of the order of $\sigma_{[B-V]^{\prime}}=0.03$ and $\sigma_{M_{v}^{\prime}}=0.1$, and the latter also includes already the typical parallax errors.

\section{Activity types versus evolution on the MS}

The strength of stellar activity, by virtue of the average $S$-value, varies continuously among solar-type stars. However, the Mt. Wilson project (Baliunas et al. 1995) has identified distinct types of temporal behaviour. Here we attempt to arrange these activity patterns in a wider context by distinguishing between (1) inactive stars (mostly by types "flat" and "long" with $S \leq 0.17)$; (2) the moderately active $(0.17 \leq S \leq 0.25)$ cases, which show two distinctly different activity forms, cyclic and irregular; and (3) the very active stars $(S \gtrsim 0.25$, always showing irregular, if not chaotic behaviour), mostly of the Mt. Wilson "var" type. We now discuss each group in detail.

\subsection{The inactive stars}

As inactive stars we consider here not only the "flat" activity stars of Baliunas et al. (1995), but also those with similarly low, near minimal (according to Schröder et al. 2012) $S$-values, accepting some low variability $(S \lesssim 0.17)$. In this fashion we have added several stars classified as "long" (term variability), as "var", or as suspected periodic (see Table 2) by Baliunas et al. (1995), and the two additional stars mentioned in Sect. 2 of yet unknown temporal behaviour. Since our focus is to find true stellar analogues of the Sun in a Maunder minimum state, this choice has proven important as is discussed below.

Six stars out of this inactive sample have been observed between 1995 and 2006 by either Lockwood et al. (2007) and/or by Hall et al. (2007) and were found to have remained inactive in those years - with one notable exception: HD 141004 increased its activity (to $\langle S\rangle=0.179$ from 0.155 during the Mt. Wilson survey, see Hall et al. 2007)! This star may have (temporarily) left its Maunder minimum state, since we would expect to observe this once in a while from our experience with the Sun, 


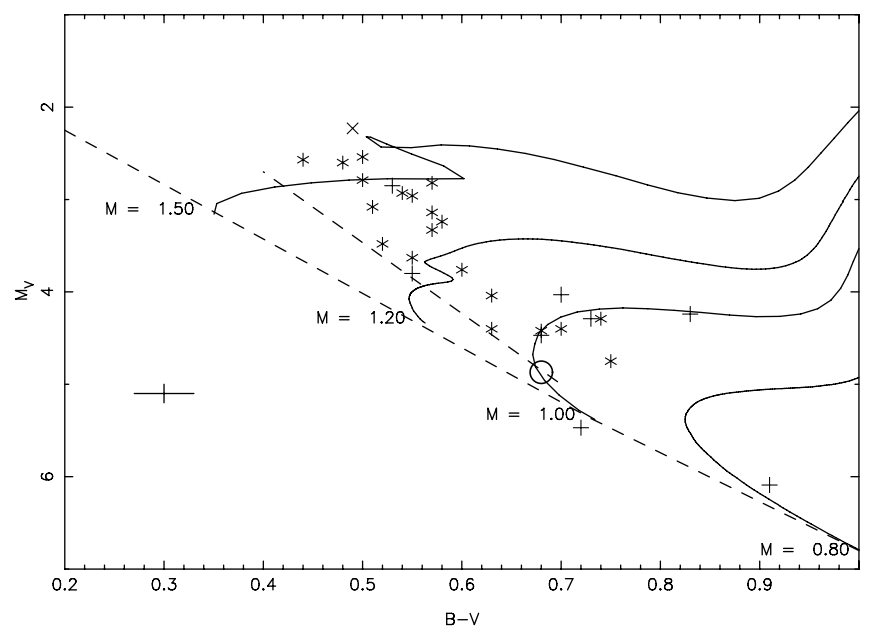

Fig. 3. Projection of all inactive stars (see text and Table 2) onto evolutionary tracks with $Z=0.02$, using the relative shifts in $B-V$ and $M_{v}$ caused by differences in metallicity between MS stars of same age and evolutionary status. Typical errors are indicated on the lower left. Metal-poor stars are marked by + , metal-rich $\eta$ Boo by $\times$, the Sun by o. Dashed lines indicate approximate positions of the ZAMS and 50\% MS lifetime. Most Mt. Wilson "flat" stars (entirely inactive) are found near the end of their MS evolution, having spent over 75\% of their MS lifetime. In contrast, a few stars not entirely inactive, and thus true equivalents of the solar Maunder minimum state, are only a bit more evolved than the Sun.

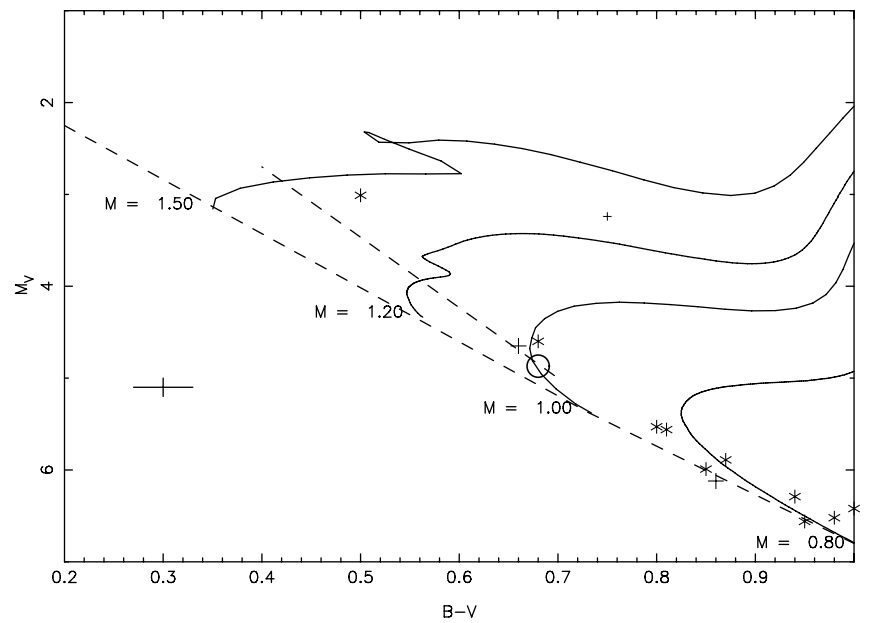

Fig. 4. Projection of Mt. Wilson stars with a sun-like, clearly developed cyclic activity (cf., Table 3 ), onto $Z=0.02$ evolutionary tracks (as in Fig. 3). Again, dashed lines indicate approximate positions of the ZAMS and 50\% MS lifetime, metal-poor stars are marked by + , the Sun by $\mathrm{o}$. This activity group dominates the 2 nd quarter of MS evolution, but a few members exceed the solar relative MS-age.

especially for stars very close to the Sun in age and mass (see below). Another interesting case is HD 43587, observed by Hall et al. (2007) at low activity levels, around $S=0.166$, while its $S$-index in the Mt. Wilson Survey was only about 0.156 , and we have recently (see Table 1 ) measured it as inactive again $(S=0.150)$.

In Fig. 3 we show the evolutionary stage and relative MS ages of these inactive stars. Even more than from Fig. 1 it is now evident that these stars are more evolved than the Sun, perhaps with two older, metal-poor exceptions, if their projection into this diagram is reliable. Most of the "flat" activity type Mt. Wilson stars are found in their final quarter of MS life, well above the approximate 50\% MS lifetime division indicated in

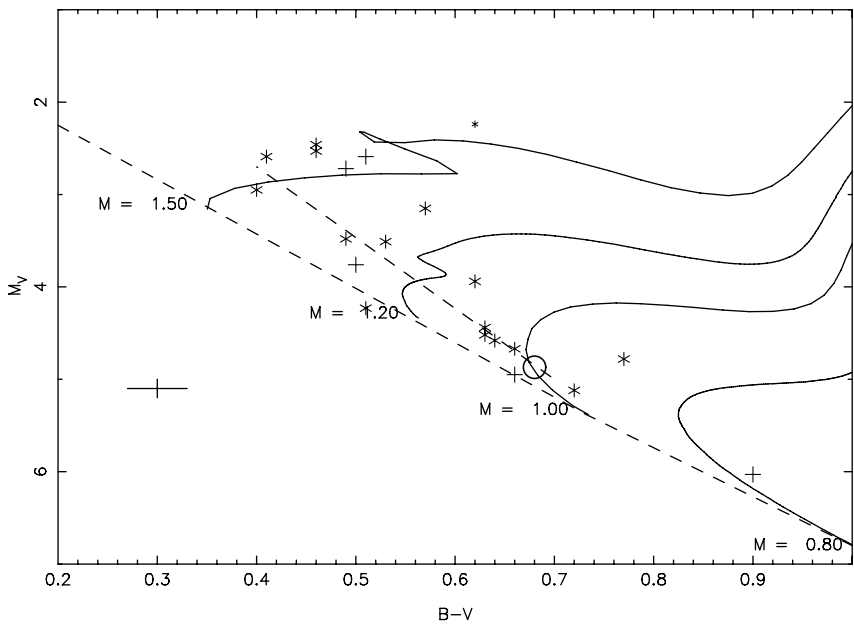

Fig. 5. HRD projection of Mt. Wilson stars with irregular or multiperiod and moderate activity $(0.17<S<0.25$, see Table 3$)$. Most of these stars are more massive than the Sun and are evolved to or beyond about half their MS lifetime. Again, dashed lines indicate approximate positions of the ZAMS and 50\% MS lifetime, metal-poor stars are marked by + , the Sun by 0 .

Fig. 3 and more closely to the turn-off side of the MS band, and have masses between 1.2 and $1.6 M_{\odot}$.

In the range of 1.0 to $1.3 M_{\odot}$ we find several stars still close to half their MS lifetime. Of these, several belong to the aforementioned group of not entirely inactive stars, classified as "long", "var", and "flat?". Considering the growing evidence from historic sunspot records that the Sun was not entirely inactive in its Maunder minimum, these stars are probably the closest analogues to that state of solar activity. Interestingly, they are all a little more evolved than the Sun. Closest to the Sun in mass and especially interesting to monitor should be HD $217014=51 \mathrm{Peg}$ ("var"), HD $146361=\sigma \mathrm{CrB}$ ("long"), HD 178428 ("flat"), HD 43587 ("long", and see above), and HD $141004=\lambda$ Ser ("long" and changes, see above). Here, 51 Peg is of particular interest, because it is known to host a planetary system. The rest of the inactive stars are either much further evolved and entirely inactive ("flat") or not a close match in mass (17 Cyg: "long", $\approx 1.3 M_{\odot}$ ), according to Fig. 3 .

At first glance, it may come as a surprise that there are hardly any inactive stars below a solar mass, in contrast to the other activity groups, especially the stars with cyclic activity. Even though such low-mass stars are, in absolute terms, older than the more massive "flat" Mt. Wilson stars, most of them turn out to be active (see below). This discovery demonstrates that it is the relative MS age, not the absolute age, which is relevant for the decay of magnetic activity among MS stars. This confirms the aforementioned, theoretical excpectations of Reiners \& Mohanty (2012).

\subsection{Moderate activity: cyclic or irregular}

Table 3 lists the clear cases of cyclic (dominated by one period, like the Sun) activity, as extracted from the Mt. Wilson project. Of these stars, we found five to have been observed in the meantime by either Lockwood et al. (2007) and/or Hall et al. (2007). In all these cases, the younger observations show an activity that is consistent with the one recorded by the Mt. Wilson campaign.

Inspecting their HRD distribution (cf., Fig. 4) we find that most of these stars have a lower mass than the Sun. All these stars appear to have a relative age similar to that of the Sun with around half of their MS lifetimes already passed, which on an evolution track would be equivalent to an advance of $\approx 1 / 3$ 
Table 4. List of the irregularly active stars of the Mt. Wilson monitoring project** $^{*}$.

\begin{tabular}{|c|c|c|c|c|c|c|c|}
\hline Star & $B-V$ & $M_{v}$ & {$[\mathrm{Fe} / \mathrm{H}]$} & {$[B-V]^{\prime}$} & $M_{v}^{\prime}$ & Type & $\langle S\rangle$ \\
\hline HD 3229 & 0.40 & 2.28 & -0.35 & 0.51 & 2.59 & poor & 0.22 \\
\hline HD 182101 & 0.40 & 3.49 & -0.31 & 0.50 & 3.76 & poor & 0.22 \\
\hline HD 61421 & 0.40 & 2.61 & +0.02 & 0.41 & 2.59 & var & 0.17 \\
\hline HD 33608 & 0.42 & 3.03 & +0.15 & 0.40 & 2.95 & long & 0.21 \\
\hline HD 176095 & 0.43 & 2.47 & -0.04 & 0.46 & 2.53 & long & 0.20 \\
\hline HD 88355 & 0.43 & 2.38 & -0.06 & 0.46 & 2.46 & var & 0.17 \\
\hline HD 2454 & 0.43 & 3.23 & -0.24 & 0.51 & 3.46 & var & 0.17 \\
\hline HD 106516 & 0.46 & 4.36 & -0.67 & 0.66 & 4.95 & var & 0.21 \\
\hline HD 157856 & 0.46 & 2.50 & -0.25 & 0.49 & 2.72 & $16 ?$ & 0.20 \\
\hline HD 16673 & 0.47 & 4.11 & -0.11 & 0.51 & 4.23 & var & 0.22 \\
\hline HD 120136 & 0.49 & 3.52 & +0.09 & 0.49 & 3.48 & poor & 0.19 \\
\hline HD 100563 & 0.53 & 3.53 & +0.06 & 0.53 & 3.51 & var & 0.20 \\
\hline HD 114378 & 0.55 & 3.76 & -0.18 & 0.62 & 3.94 & long & 0.24 \\
\hline HD $6920^{*}$ & 0.56 & 2.09 & -0.14 & 0.62 & 2.24 & var & 0.19 \\
\hline HD 176051 & 0.57 & 4.39 & -0.19 & 0.64 & 4.58 & ? & 0.18 \\
\hline HD 100180 & 0.57 & 4.36 & -0.15 & 0.63 & 4.52 & $3.6 ?$ & 0.17 \\
\hline HD 137107 & 0.58 & 4.32 & -0.10 & 0.63 & 4.44 & var & 0.18 \\
\hline HD 12235 & 0.60 & 3.27 & +0.18 & 0.57 & 3.15 & var & 0.16 \\
\hline HD 190406 & 0.61 & 4.55 & -0.12 & 0.66 & 4.67 & $17+3$ & 0.19 \\
\hline HD 224930 & 0.67 & 5.32 & $-0.78^{+}$ & 0.90 & 6.03 & poor & 0.18 \\
\hline HD 115617 & 0.70 & 5.08 & +0.00 & 0.72 & 5.12 & var & 0.16 \\
\hline HD 3443 & 0.71 & 4.63 & -0.14 & 0.77 & 4.78 & var & 0.18 \\
\hline HD 111456 & 0.43 & 3.94 & -0.19 & 0.50 & 4.13 & $?$ & 0.30 \\
\hline HD 25998 & 0.47 & 3.89 & +0.02 & 0.48 & 3.91 & long & 0.30 \\
\hline HD 75332 & 0.50 & 3.96 & +0.02 & 0.51 & 3.98 & var & 0.28 \\
\hline HD 35296 & 0.52 & 4.21 & -0.15 & 0.58 & 4.37 & long & 0.33 \\
\hline HD 26923 & 0.56 & 4.66 & -0.13 & 0.61 & 4.81 & var & 0.28 \\
\hline HD 206860 & 0.58 & 4.69 & -0.19 & 0.65 & 4.89 & poor & 0.33 \\
\hline HD 115043 & 0.58 & 4.80 & -0.22 & 0.66 & 5.02 & var & 0.32 \\
\hline HD 154417 & 0.58 & 4.43 & -0.08 & 0.62 & 4.53 & $7 ?$ & 0.27 \\
\hline HD 72905 & 0.58 & 4.87 & -0.25 & 0.66 & 5.12 & var & 0.35 \\
\hline HD 115383 & 0.59 & 4.00 & +0.03 & 0.60 & 4.00 & var & 0.32 \\
\hline HD 12933 & 0.59 & 4.94 & $\mathrm{n} / \mathrm{a}$ & 0.61 & 4.97 & var & 0.55 \\
\hline HD 39587 & 0.60 & 4.71 & -0.16 & 0.66 & 4.88 & var & 0.33 \\
\hline HD 97334 & 0.61 & 4.71 & -0.09 & 0.66 & 4.82 & var & 0.33 \\
\hline HD 30495 & 0.64 & 4.88 & -0.08 & 0.68 & 4.98 & var & 0.30 \\
\hline HD 26913 & 0.66 & 5.30 & -0.17 & 0.73 & 5.48 & $8 ?$ & 0.40 \\
\hline HD 20630 & 0.66 & 5.05 & 0.00 & 0.68 & 5.08 & $6 ?$ & 0.37 \\
\hline HD 1835 & 0.67 & 4.78 & -0.02 & 0.70 & 4.83 & $9 ?$ & 0.35 \\
\hline HD131156A & 0.68 & 5.46 & $\mathrm{n} / \mathrm{a}$ & 0.70 & 5.49 & var & 0.48 \\
\hline HD 101501 & 0.73 & 5.41 & -0.12 & 0.78 & 5.55 & var & 0.33 \\
\hline HD 82885 & 0.77 & 5.06 & $\mathrm{n} / \mathrm{a}$ & 0.79 & 5.09 & $8 ?$ & 0.28 \\
\hline HD 82443 & 0.77 & 5.76 & $\mathrm{n} / \mathrm{a}$ & 0.79 & 5.78 & poor & 0.64 \\
\hline HD 165341A & 0.78 & 5.67 & -0.29 & 0.87 & 5.96 & $5 ?$ & 0.39 \\
\hline HD 149661 & 0.80 & 5.82 & -0.01 & 0.82 & 5.86 & $17+4$ & 0.34 \\
\hline HD 10780 & 0.81 & 5.62 & +0.05 & 0.82 & 5.62 & ? & 0.28 \\
\hline HD 37394 & 0.84 & 5.78 & -0.02 & 0.87 & 5.83 & poor & 0.45 \\
\hline HD 155886 & 0.85 & 6.39 & $\mathrm{n} / \mathrm{a}$ & 0.87 & 6.41 & poor & 0.38 \\
\hline HD 155885 & 0.85 & 6.19 & $\mathrm{n} / \mathrm{a}$ & 0.87 & 6.21 & var & 0.39 \\
\hline HD 22049 & 0.88 & 6.19 & 0.00 & 0.90 & 6.21 & var & 0.50 \\
\hline HD 17925 & 0.91 & 5.92 & +0.03 & 0.92 & 5.92 & long & 0.65 \\
\hline
\end{tabular}

Notes. ${ }^{(*)}$ Post-MS star +) Pop.II? ${ }^{(* *)}$ Moderately active $(0.17<$ $S<0.25$, above) and strongly active $(S>0.25$, below) stars, each group organized by observed $B-V$, activity strength is indicated by the average Mt. Wilson $S$-value, periods are given in years in the column "type", according to Baliunas et al. (1995).

from the starting point on the zero age main sequence (ZAMS) to the turn-off point. Considering the uncertainties in the projected HRD positions discussed above, and especially the fact that lower mass stars' tracks run parallel to the ZAMS, we cannot quantify this any further.

Nevertheless, the stars with well developed cycles represent only a smaller fraction of the active Mt. Wilson stars: what kind of activity does characterize the others? Looking at the other moderately active Mt. Wilson stars $(0.17<S<0.25$, see Table 4, upper section), we note that most of these are more massive than the Sun. There appears to be an empirical upper mass-limit for MS stars showing a cyclic activity behaviour, not much above one solar mass.

Figure 5 shows that most of the multi-periodically or chaotically variable stars of moderate activity have (like their less massive, cyclic counterparts) reached about half their MS lifetime. Only, many irregular moderate-active stars are a bit older, and therefore, this distribution shows a gradual transition further towards the third quarter of MS lifetime, and further towards the more evolved and already inactive stars (compare Fig. 5 with Fig. 3).

We note that in four cases, the irregular, moderate activity of these stars was confirmed by either Lockwood et al. (2007) and/or Hall et al. (2007) during the 15 years following the Mt. Wilson project. Some of the non-cyclic stars come close to the Sun in mass and relative age (halfway through their MS life), as well as by average $S$-value (around 0.18): HD 100180, HD 137107, HD 176051, HD 190406. In two of these cases, activity shows a superposition of two very distinct periods. The exact nature of activity in these four stars should be of great interest for better understanding the solar dynamo.

\subsection{Strong activity: The irregular stars}

In Fig. 6 we finally present the subsample of highly active stars ( $S>0.25$, see Table 4, lower section), which are all of irregular and more or less chaotic variability. In 16 cases, this nature was confirmed by either Lockwood et al. (2007) and/or Hall et al. (2007) during another 15 years following the Mt. Wilson project. In addition, many of these stars are known to be variable even in broadband photometry. Comparing Fig. 6 with Fig. 4, it appears that this stellar sample's average evolutionary state is very young, with the most active stars $(S>0.35$, large symbols) scattered around the ZAMS itself. The bulk of the remaining stars are much less evolved than the Sun. Again, for the reasons given above, it is impossible to quantify this any better.

It is therefore expected that all "young" (that is: relative to their MS lifetime) Mt. Wilson stars show high and irregular activity. Stars less massive than the Sun then behave like the latter and become cyclic, once their activity has moderated. The more massive Mt. Wilson stars still fail to settle into such a singleperiod mode and continue with irregular activity until they become mostly inactive in the last quarter of their MS life.

\section{Discussion and conclusions}

A comparison of Figs. 3-5 shows that the stars in the different activity groups populate different parts of the MS band. Unfortunately, it is not possible to individually quantify the relative ages of the early, still quite slowly evolving MS stars, but we can nevertheless make a strong qualitative observation. There is a good correlation of activity strength with relative MS-age, rather than with absolute age, in good agreement with recent theoretical expectation (Reiners \& Mohanty 2012).

The Sun's relative MS age is typical of those stars with a clearly defined cyclic activity, its next (3rd) quarter of MSevolution will lead the Sun through the domain of Maunder minimum analogues (almost inactive stars). In that sense, the historical solar Maunder minimum must appear as a precursor of what lies ahead as the solar activity cycle is nearing its lower stability limit. It already goes faint occasionally and will gradually do so 


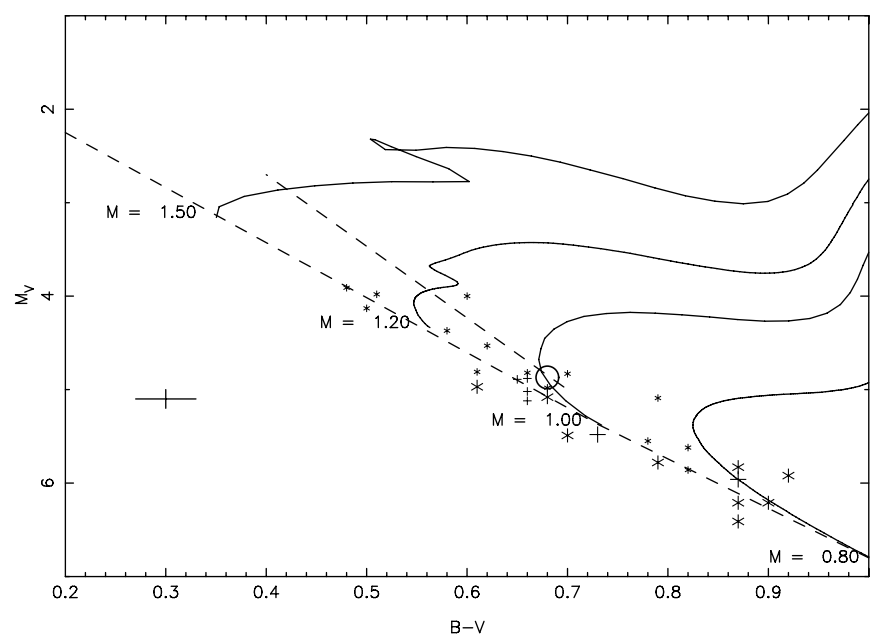

Fig. 6. HRD projection of the most active Mt. Wilson stars (with $S=$ $0.4 \ldots 0.5$ and "var" type, see large symbols); these stars are found very close to the ZAMS. Again, dashed lines indicate approximate positions of the ZAMS and 50\% MS lifetime, metal-poor stars are marked by + , the Sun by o. Most other more active stars $(S>0.25$, see Table 4$)$ are still much less evolved than the Sun.

more often in the next 1-2 billion years until, in its final quarter of MS life, the Sun will become entirely inactive ("flat").

On the other hand, the Sun also lies near the mass range, where we find a transition from predominantly cyclic activity (lower mass) to predominantly irregular or multi-periodic activity (higher mass) among the moderately evolved and active stars. This adds to the interpretation that the Sun's dynamo may be operating near its lower stability limit. For the more massive stars ( 1.2 to $1.5 M_{\odot}$ ), the question as to how their activity actually works and why it is not cyclic. Since these stars have a thinner convective envelope than the Sun, we may speculate that only a sufficiently deep convection zone (as for low mass stars) allows the dynamo to settle into its mono-periodic fundamental mode, when activity has calmed down a bit.

The Mt. Wilson sample is quite small and not complete. In terms of population density, therefore we can only argue in a qualitative way. Nevertheless, it is clear that the large majority of stars in their final quarter of MS life has become entirely inactive ("flat"), as already pointed out by Wright (2004). There are perhaps only two such late-MS cyclic stars (Fig. 4) and half a dozen non-cyclic, barely active stars, but two or three of these may be discounted as already post-MS. Compared with the about 20 inactive stars, it follows that over $70 \%$ of all late-MS stars have become entirely inactive.

This finding, however, contrasts to the large number of giants, in the same moderate mass range considered here, that still show activity (Hünsch et al. 1996; Hünsch \& Schröder 1996) in the form of coronal X-ray emission above the minimal coronal X-ray flux of Schmitt (1997). This makes the one or two cases of activity found high up on the RGB, and perhaps even AGB, as recently reported by Konstantinova-Antova et al. (2010), even stranger. They directly measured magnetic fields of several Gauss in the photospheres of several $\mathrm{M}$ giants selected for large coronal X-ray flux and fast rotation.

The consistent decrease of stellar activity with MS-evolution shown here implies that magnetic activity must be turned on again in the course of post-MS evolution. Indeed, first Kepler g-mode observations have now provided direct evidence that the core of a giant star rotates more than ten times faster than its envelope (Beck et al. 2012). The core region is thus keeping some of its angular momentum into post-MS evolution, and its contraction leads to a spin-up, thus providing a fresh source of differential rotation. Is the resulting dynamo the same kind as the one we observe in solar-type MS stars? Only larger samples of well-characterized stars, with close solar matches of different age, including giants, will give answers to these questions.

Acknowledgements. We gratefully acknowledge the efforts of decades of devotion to the Mt. Wilson stellar activity monitoring project, without which our study would have been impossible. The remarks of an anonymous referee were very useful and improved our paper, and we very much appreciate the important input of Jeffrey Hall. In addition, we kindly acknowledge funding support from the bilateral (MEX-GER) Conacyt-DFG project No. 147902, as well as the use of the Strasbourg data bases, the HIPPARCos parallaxes, and the financial support for the Hamburg Robotic Telescope project given by the Universities of Hamburg, Guanajuato, and Liege, and by the state of Guanajuato.

\section{References}

Baliunas, S. L., Donahue, R. A., Soon, W. H., et al. 1995, ApJ, 438, 269 Baliunas, S. L., Donahue, R. A., Soon, W., \& Henry, G. W. 1998, Cool Stars, Stellar Systems, and the Sun, 154, 153

Beck, P., Montalban, J., Kallinger, T., et al. 2012, Nature, 481, 55

Duncan, D. K., Vaughan, A. H., Wilson, O. C., et al. 1991, ApJS, 76, 383

Eddy, J. A. 1976, Science 192, 1189

Fröhlich, C. 2011, Space Sci. Rev., DOI: 10.1007/s11214-011-9780-1

Hall, J. C., \& Lockwood, G. W. 2004, ApJ 614, 942

Hall, J. C., Lockwood G. W., \& Skiff, B. A. 2007, ApJ, 133, 862

Hempelmann, A., \& Weber, W. 2011, Sol. Phys., DOI: 10.1007 s1 1207-011-9905-4

Hempelmann, A., Schmitt, J. H. M. M., \& Stȩpieǹ, K. 1996, A\&A, 305, 284

Hempelmann, A., González-Pérez, J. N., Schmitt, J. H. M. M., \& Hagen, H. J. 2005, in Cool Stars, Stellar Systems, and the Sun 13, eds. F. Favata, G. A. J. Hussain, \& B. Battrick, ESA SP 560, 643

Holmberg, J., Nordström, B., \& Andersen, J. 2009, A\&A, 501, A94

Hünsch, M., \& Schröder, K.-P. 1996, A\&A, 309, L51

Hünsch, M., Schmitt, J. H. M. M., Schröder, K.-P., \& Reimers, D. 1996, A\&A, 310, 801

Ineson, S., Scaife, A. A., Knight, J. R., et al. 2011, Nature Geosc. Lett., DOI: $10.1038 /$ NGE01282

Konstantinova-Antova, R., Aurière, M., Charbonnel, C., et al. 2010, A\&A, 524, A57

Lockwood, G. W., Skiff, B. A., Henry, G. W., et al. 2007, ApJS, 171, 260

Mittag, M., Hempelmann, A., González-Pérez, J. N., \& Schmitt, J. H. M. M. 2010, Adv. Astron., 2010, 101502

Nagovitsyn, Yu. A. 2007, Astron. Lett., 33, 340

Pallavicini, R., Golub, L., Rosner, R., et al. 1981, ApJ, 248, 279

Pérez Martínez, M. I., Schröder, K.-P., \& Cuntz, M. 2011, MNRAS, 414, 418

Perryman, M. A. C. 2002, Ap\&SS, 280, 1

Pietrinferni, A., Cassisi, S., Salaris, M., \& Castelli, F. 2004, ApJ, 612, 168

Pietrinferni, A., Cassisi, S., Salaris, M., \& Castelli, F. 2006, ApJ, 642, 797

Pols, O. R., Tout, C. A., Schröder, K.-P., Eggleton, P. P., \& Manners, J. 1997, MNRAS, 289, 869

Pols, O. R., Schröder, K.-P., Hurley, J. R., Tout, C. A., \& Eggleton, P. P. 1998, MNRAS, 298, 525

Reiners, A., \& Mohanty, S. 2012, ApJ, 746, 43

Roxburgh, I. W. 1992, A\&A, 266, 291

Schmitt, J. H. M. M. 1997, A\&A, 318, 215

Schrijver, C. J. 1987, A\&A, 172, 111

Schröder, K.-P., \& Pagel, B. E. J. 2003, MNRAS, 343, 1231

Schröder, K.-P., Pols, O. R., \& Eggleton, P. P. 1997, MNRAS, 285, 696

Schröder, K.-P., Mittag, M., Pérez Martínez, M. I., Cuntz, M., \& Schmitt, J. H. M. M. 2012, A\&A, 540, A130

Simon, T. 1990, ApJ, 359, L51

Simon, T. 1992, in 7th Cambridge Workshop on Cool Stars, Stellar Systems, and the Sun, eds. M. S. Giampapa, \& J. A. Bookbinder (San Francisco), ASP Conf. Proc., 26, 3

Skumancich, A. 1972, ApJ, 171, 565

Stauffer, J. R., Hartmann, L., Soderblom, D. R., \& Burnham, N. 1984, ApJ, 280, 202

Stauffer, J. R., Hartmann, L. W., Burnham, J. N., \& Jones, B. F. 1985, ApJ, 289, 247

Strassmeier, K. G., Handler, G., Paunzen, E., \& Rauth, M. 1994, A\&A, 281, 855

Svensmark, H. 1998, Phys. Rev. Lett., 81, 5027

Weber, W. 2010, Ann. Phys., 522, 372

Weiss, N. O. 2010, A\&G, 51, 3.09

Wright, J. T. 2004, ApJ, 128, 1273

Wright, J. T., Marcy, G. W., Butler, R. P., \& Vogt, S. S. 2004, ApJS, 152, 261 